\title{
Unfalsified Adaptive Control for manipulators with parameter uncertainties
}

\author{
Jaime Arango*, Carlos Ocampo-Martinez ${ }^{\dagger}$, Fernando Bianchi ${ }^{\ddagger}$ and Gustavo Osorio* \\ ${ }^{*}$ Department of Electrical and Electronic Engineerings and Computer Science \\ Universidad Nacional de Colombia, Km 9 Via Aeropuerto La Nubia \\ Manizales, Colombia \\ Email: [jearangoca,gaosoriol]@unal.edu.co \\ †Institut de Robòtica i Informàtica Industrial (CSIC-UPC) \\ Universitat Politècnica de Catalunya - Barcelona Tech, Llorens i Artigas, 4-6, 08028 \\ Barcelona, Spain. \\ Email: cocampo@iri.upc.edu \\ $\ddagger$ Catalonia Institute for Energy Research, IREC, \\ Jardins de les Dones de Negre 1, 08930 Sant Adrià de Besòs \\ Barcelona, Spain. \\ Email: fbianchi@irec.cat
}

\begin{abstract}
This work evaluates by simulation the performance of the Unfalsified Adaptive Control (UAC) for Multiple Degree of Freedom (MDoF) serial manipulators. The UAC is a data-driven technique that addresses stability issues of model-based controllers for robot arms with inertial uncertainties. The unfalsified controller selects the most suitable controller from a set, based on performance, to decide whether the controller in the closed loop should be changed, using only system inputs and outputs, i.e., torques and joint variables of the robotic arm, respectively. In this work, performance and robustness is evaluated by simulation on a 5-DoF manipulator showing the ability of the UAC to accomplish tracking tasks in the presence of inertial parameters disturbances.
\end{abstract}

\section{INTRODUCTION}

For the last decades, both model-based and data-driven control techniques has been used for manipulators. Traditional model-based strategies are often applied dealing with imprecise model identification or measurement of inertial and friction parameters [1], [2], [3], [4]. But, the identification or measurement of parameters is a demanding task, with difficulties on excitation functions, validation of results [5], [6], [7], [8] and possible unexpected performance of the plant [5]. Due to the increasing complexity of robot arm applications like lifting or lowering objects, picking up objects from shelves or helping people with personal care activities, careful evaluation of control performance in novel approaches becomes an issue of interest, considering the analysis of the ability to handle payloads in common daily situations due to sudden changes or unstructured environments [9], [10], [11].

The Unfalsified Adaptive Control (UAC or UC) theory is a data-driven approach in the field of adaptive control [12], [13], [14]. The main advantage with respect to other techniques relies on its independence of the system model, with reliance only on the measurement of inputs and outputs of the plant (the robotic manipulator in this case). The basic elements of an UAC controller are a set of stabilizing controllers, a performance criterion and a switching strategy. The performance of each controller within a set is evaluated outside the closed loop by using measures of system inputs and outputs. If any tested controller shows a better performance, a switching strategy conveniently replaces the controller in the loop. The UAC technique has been proposed for the control of 2-DoF planar robotic manipulators [13], [15], [14], using linear programing methods for parameter adaption. Unfortunately, the internal stability of the closed-loop system provided in [15] is not fully formal. Moreover, in [16] and [17] cases of instability are analyzed and in [18] simple stability tests are proposed for closed-loop stability. The use of UAC for more complex manipulators is studied by simulation in [19], based on the proposed methods in [15]. In particular, the authors analyzed performance of specific manipulators with bounded disturbances. Recent works present applications of UAC for fuel cell systems [20], secondary mirror positioning control for ground-based telescopes [21], inertial navigation systems [22], pressure drilling for oil [23] [24], temperature tracking of the Chylla-Haase reactor [25] and inverted pendulum systems [26].

In this work, we evaluate in simulation both performance and robustness of the UAC technique for MDoF manipulators, considering the effects due to inertial parameters disturbances and payload changes. To this end, it is used the traditional model-based computed torque technique to design the candidate controller set. The used UAC strategy includes fading memory introduced in [27] to emphasize the significance of new data in the performance evaluation, improving the ability to detect destabilizing controllers. Three situations for performance evaluation have been considered: two fixed payloads, periodic non-smooth payload variations and worstcase disturbances to the inertial parameters to test robustness. The UAC controller has shown acceptable performance and low dependency to inertial parameters perturbations.

The remainder of the paper is organized as follows: Section II introduces UAC definitions, basic concepts and the 
application to MDoF manipulators. In Section III, the UAC controller is evaluated in simulation using a 5-DoF manipulator dynamical model. Finally, the main conclusions are drawn in Section IV.

\section{UNFALSIFIED ADAPTIVE CONTROL FOR MDOF MANIPULATORS}

The UAC is inspired by [28] standing that learning is achieved by using experimental data to falsify hypotheses. To this end, three sets are defined: $\mathbf{P}$ of signals $(\mathbf{r}, \mathbf{u}, \mathbf{y})$ related with past measurements of inputs $\mathbf{u}$, outputs $\mathbf{y}$ and the reference $\mathbf{r} ; \mathbf{M}$ of signals $(\mathbf{r}, \mathbf{u}, \mathbf{y})$ related to the behavior of the controller $K$ in the closed loop; and $\mathbf{T}$ of signals $(\mathbf{r}, \mathbf{u}, \mathbf{y})$ that accomplish the expected performance [14].

Then, the controller $K$ is not falsified if the intersection between the set of past measurements of inputs, outputs and reference signals and the set of controller $\mathbf{K}$ signals is a subset of the performance evaluation signals $(\mathbf{P} \cap \mathbf{M} \subset \mathbf{T})$, meaning that the controller stays in the loop since it has the best performance compared with a set of different controllers. Otherwise, the controller in the loop is falsified and changed.

The main advantage of UAC as a supervisory robust controller is that a model of the plant is not necessary [29], [13], [14]. The controller in the loop can be falsified or not just with the information given by the input and output data $\mathbf{z}=(\mathbf{u}, \mathbf{y})$. Besides, this may be applied to the controllers outside the loop. To this end, the controllers should be causally left invertible, i.e., it should be possible to compute a fictitious reference signal $\tilde{\mathbf{r}}$ from the inversion of the controller expression and the data $\mathbf{z}$ (see pag. 20 of [14]).

The performance criterion for the UAC strategy is based on a cost function capable of detecting from the data $\mathbf{z}$ whether or not the controller is stabilizing. The cost-detectable function used here is presented in [27] and justified in [14], which takes advantage of the fading factor to reduce the probability of a large transient and is bounded if there exists at least one stabilizing candidate controller in the set. The cost function is defined as

$$
V(K, \mathbf{z}, t)=\frac{F_{\eta}(e(t), t)+F_{\eta}(u(t), t)}{F_{\eta}(\tilde{r}(t), t)+\alpha},
$$

where $e(t)=\tilde{r}(t)-y(t), \alpha>0$ prevents division by zero and

$$
F_{\eta}(y(t), t)=\sum_{\delta=0}^{t} y^{T}(\delta) y(\delta) \eta^{t-\delta},
$$

where $\delta$ is the evaluation time period, $\eta<1$ is a fading factor to emphasize the significance of new data. This function allows the UAC algorithm to select which controller has the best performance based on current operating conditions [27].

The UAC technique may use any control theory to design a finite controller set $\mathbf{K}=\left\{K_{i}, i=1, \ldots, N\right\}$, and a switching algorithm that, at each time $t_{k}$, verifies

$$
\min _{i}\left(V\left(K_{i}, \mathbf{z}, t_{k}\right)\right)<V\left(\hat{K}, \mathbf{z}, t_{k-1}\right)+\varepsilon
$$

where $\hat{K} \in \mathbf{K}$ is the controller in the closed-loop at $t_{k-1}$ and $\varepsilon$ is a small hysteresis constant. If the condition (3) holds, the current controller $\hat{K}$ is replaced by $K_{i} \in \mathbf{K}$ [30].

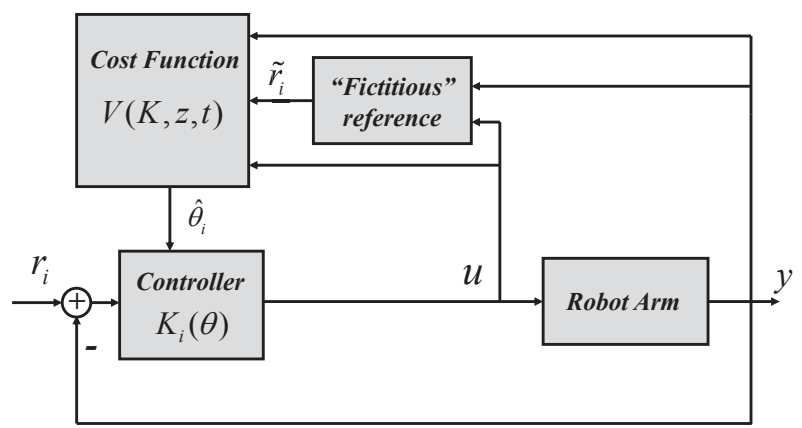

Fig. 1: Unfalsified Adaptive Control for multiple DoF robotic arms

In the UAC scheme for MDoF manipulators of Fig. 1, the fictitious reference set $\tilde{\mathbf{r}}$ is computed from the control signal $\mathbf{u}$ or torques required at joints, and output positions, velocities and accelerations as the data $\mathbf{y}$, for each controller $K_{i} \in \mathbf{K}$.

The dynamical model of a $n$-link rigid and non-flexible joints manipulator (robot arm), based on traditional mechanics of Newton-Euler or Lagrange-Euler, are highly nonlinear and might be formulated as

$$
\mathbf{H}(\theta, \mathbf{q}) \ddot{\mathbf{q}}+\mathbf{C}(\theta, \mathbf{q}, \dot{\mathbf{q}}) \dot{\mathbf{q}}+\mathbf{G}(\theta, \mathbf{q})+\mathbf{F}(\dot{\mathbf{q}})=\mathbf{u},
$$

where $\theta$ is a parameter vector, function of the inertial terms; $\mathbf{q}, \dot{\mathbf{q}}, \ddot{\mathbf{q}} \in \mathbb{R}^{n}$ are position, velocity and acceleration joint vectors, respectively. $\mathbf{H}(\theta, \mathbf{q})$ is the $n \times n$ inertia matrix, $\mathbf{C}(\theta, \mathbf{q}, \dot{\mathbf{q}}) \dot{\mathbf{q}}$ contains the $n \times 1$ vector of Coriolis and centripetal terms, $\mathbf{G}(\boldsymbol{\theta}, \mathbf{q})$ is the $n \times 1$ vector of gravitational terms, $\mathbf{F}(\dot{\mathbf{q}})$ is the $n \times 1$ vector of viscous friction and $\mathbf{u}$ is the $n \times 1$ vector of torques applied to the joints of the manipulator.

As a function of a parameter vector $\theta$, the model may be presented in the form of

$$
\mathbf{Y}(\mathbf{q}, \dot{\mathbf{q}}, \dot{\mathbf{q}}, \ddot{\mathbf{q}}) \theta+\mathbf{G}(\theta, \mathbf{q})+\mathbf{F}(\dot{\mathbf{q}})=\mathbf{u} .
$$

Therefore, the dynamical model is linear with respect to the parameter vector $\theta$ for any $n$ degree of freedom manipulator.

The UAC algorithm checks every sampling time $T_{s}$ the condition (3) and decides whether or not the controller in the loop should be replaced. One disadvantage of this procedure might be a computational inefficiency when the number of candidate controllers is large due to the calculation of the fictitious reference for each controller in the set.

The reference $\mathbf{r}$, input torques $\mathbf{u}$ and output positions, velocities and accelerations $\mathbf{y}$ are defined as

$$
\begin{aligned}
\mathbf{r} & =\left[\begin{array}{lll}
\mathbf{q}_{\mathbf{d}}^{T} & \dot{\mathbf{q}}_{\mathbf{d}}^{T} & \ddot{\mathbf{q}}_{\mathbf{d}}^{T}
\end{array}\right]^{T} \in \mathbb{R}^{3 n}, \\
\mathbf{u} & =\tau \in \mathbb{R}^{n}, \\
\mathbf{y} & =\left[\begin{array}{lll}
\mathbf{q}^{T} & \dot{\mathbf{q}}^{T} & \ddot{\mathbf{q}}^{T}
\end{array}\right]^{T} \in \mathbb{R}^{3 n} .
\end{aligned}
$$

The fictitious reference

$$
\tilde{\mathbf{r}}_{\mathbf{i}}=\left[\begin{array}{lll}
\hat{\mathbf{q}}_{\mathbf{d}}^{T} & \dot{\hat{\mathbf{q}}}_{\mathbf{d}}{ }^{T} & \ddot{\hat{\mathbf{q}}}_{\mathbf{d}}^{T}
\end{array}\right]^{T} \in \mathbb{R}^{3 n}
$$

may be easily computed from the inversion of the candidate controller. In this case, with the traditional computed torque 
controller and using the measured data $\mathbf{z}$, the fictitious reference may be obtained from

$$
\begin{aligned}
& \ddot{\hat{\mathbf{q}}}_{\mathbf{d}}+2 \lambda \dot{\hat{\mathbf{q}}}_{\mathbf{d}}+\lambda^{2} \hat{\mathbf{q}}_{\mathbf{d}}=\mathbf{H}^{-1}\left(\tau+\mathbf{H}\left(\ddot{\mathbf{q}}+2 \lambda \dot{\mathbf{q}}+\lambda^{2} \mathbf{q}\right)\right. \\
& -\mathbf{H} \ddot{\mathbf{q}}-\mathbf{C} \dot{\mathbf{q}}-\mathbf{G}-\mathbf{F}) .
\end{aligned}
$$

\section{ANALYSIS OF PERFORMANCE AND ROBUSTNESS}

In this section we evaluate in simulation both performance and robustness of the UAC technique for MDoF manipulators, considering the effects due to inertial parameters disturbances and payload changes. To this end, it is used the traditional model-based computed torque technique to design the candidate controller set of the UAC. The cost-detectable function of the UAC includes fading memory, as presented in Section II, to emphasize the significance of new data in the performance evaluation, improving the ability to detect destabilizing controllers. Three situations for performance evaluation have been considered: two fixed payloads, periodic non-smooth payload variations and worst-case disturbances to the inertial parameters to test robustness.

The case of study is a 5-DoF rigid manipulator, with the parameters presented in [31]:

$$
\begin{aligned}
m_{i} & =1 \mathrm{~kg}, i=1, \ldots, 5, \\
l_{1} & =0.35 \mathrm{~m}, l_{2}=l_{3}=0.22 \mathrm{~m}, l_{4}=l_{5}=0.14 \mathrm{~m}, \\
\gamma_{i} & =0.1 \mathrm{~N} \mathrm{~s} / \mathrm{m}, i=1, \ldots, 5, \text { (Damping friction) } \\
\mathbf{I}_{\mathbf{x y}} & = \begin{cases}0.1, & \text { if } x=1, \ldots, 5 \text { and } y=1,2,3, \\
0, & \text { if } x=1, \ldots, 5 \text { and } y=4,5 .\end{cases}
\end{aligned}
$$

This model was used in [32] to analyze performance with traditional control techniques. Several simulations showed a maximum payload capacity of the manipulator for the tracking task of about $2 \mathrm{~kg}$. The payload is added to the last element of the manipulator, with mass $m_{5}$, and is modified in the following scenarios for analysis.

Periodic articular trajectories with period $T=5 \mathrm{~s}$ are used for performance analysis:

$$
q_{d_{i}}= \begin{cases}\frac{2 \pi}{3}\left(1-\cos \left(\frac{2 \pi}{5} t\right)\right), & \text { if } i=1,3,5, \\ \pi\left(1-\cos \left(\frac{2 \pi}{5} t\right)\right), & \text { if } i=2,4\end{cases}
$$

Simulations started at rest, from initial conditions: $q_{d_{i}}(0)=0$ and $\dot{q}_{d_{i}}(0)=0$, with $i=1, \ldots, 5$. Saturation torques of actuators are assumed constant for simplicity at $\pm 30 \mathrm{Nm}$.

We used the traditional computed torque controller for the controller set $\mathbf{K}$ related to the UAC, with known inertial parameters and $\lambda=20$ in (8), with stable critically damped behavior as presented in [32], assuring feasibility for the stability of the UAC, as requested in [33]. The parameter $\theta$ (the sum of the masses of the end-effector and the payload) takes finite values $\hat{\theta}$ in a set $\Theta$, taking advantage of the a priori knowledge of the payload interval, ranging from the no-load condition to the maximum payload of the manipulator in the workspace, i.e.,

$$
\Theta=\{1,2,3\}
$$

Considering that $m_{5}=1 \mathrm{~kg}$ (the constant mass of the end effector without payload), the interval for the controller parameter (payload) is $[0,2] \mathrm{kg}$, with the upper limit of the interval corresponding to the maximum payload supported by the manipulator. Three values are used for the set $\Theta$, based on the payload interval of the manipulator, to reduce the on-line computation time of control signals and fictitious references.

The cost function constants in (1) are: $\alpha=0.001$ and $\eta=0.9$ as the fading constant. This function is evaluated periodically with $T_{s}=0.01 \mathrm{~s}$, as stated in the procedure described in Section II.

The performance of the UAC was evaluated for three different cases: $i$ ) performance of the controller for two fixed payloads; ii) performance of the controller with periodic sudden payload changes; and iii) performance with worst-case disturbances in the inertial parameters.

We used control effort (CE) and integral squared tracking error (ISE) as performance evaluation indexes, considering their relation to the cost-detectable function in (1). We used a moving window for the computation of CE and ISE integrals for the entire period of the desired trajectories $(T=5 \mathrm{~s}$ in this case), defining the integration interval between $t-T$ and $t$. The ISE is computed as

$$
I S E_{q_{i}}(t)=\int_{t-T}^{t} e_{i}^{2}(t) d t,
$$

where $e_{i}(t)(i=1, \ldots, 5)$ are the tracking errors.

The CE is computed as

$$
C E_{q_{i}}(t)=\int_{t-T}^{t} \tau_{i}^{2}(t) d t,
$$

where $\tau_{i}(t)(i=1, \ldots, 5)$ are the control torques. Note that although these calculations are presented in continuous time, in practice they are computed in discrete time, with relatively small sampling time compared with the evaluation of the cost functions in (3).

\section{A. Controller performance analysis for two fixed payloads}

Simulations are performed for two constant payloads along the trajectory, with $1.5 \mathrm{~kg}$ and $1.7 \mathrm{~kg}$, and without disturbances to the inertial parameters.

Figure 2 shows periodic errors that increase at the beginning of each period due to the most effort demanding points of trajectories for joints 2 and 3. It shows a performance loss at those points related to the increased payload, but the ability of the system to recover for an acceptable performance. The system with the UAC tracks the desired trajectories.

Control signals for both payloads are presented in Fig. 3. For joint $q_{2}$, the controller recovers from saturation in the most effort demanding points. Short transients for joint $q_{3}$ occur at these same points of the testing trajectories. As shown in Fig. 4, a change of the controller parameter appears at these effort demanding points, generating those transients. It also should be noted from Fig. 4 that one period (from $5 \mathrm{~s}$ to 10 s) of periodic cost functions shows a finite number of changes of the controller parameter (right side of the Fig. 4). 

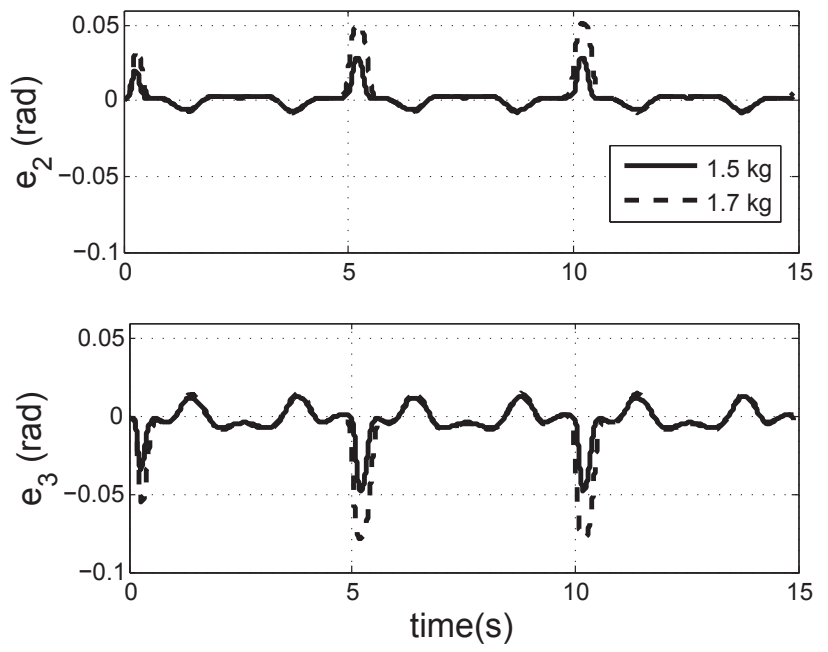

Fig. 2: Comparison of tracking errors for $q_{2}$ and $q_{3}$. Solid line for Payload of $1.5 \mathrm{~kg}$. Dashed line for Payload of $1.7 \mathrm{~kg}$.
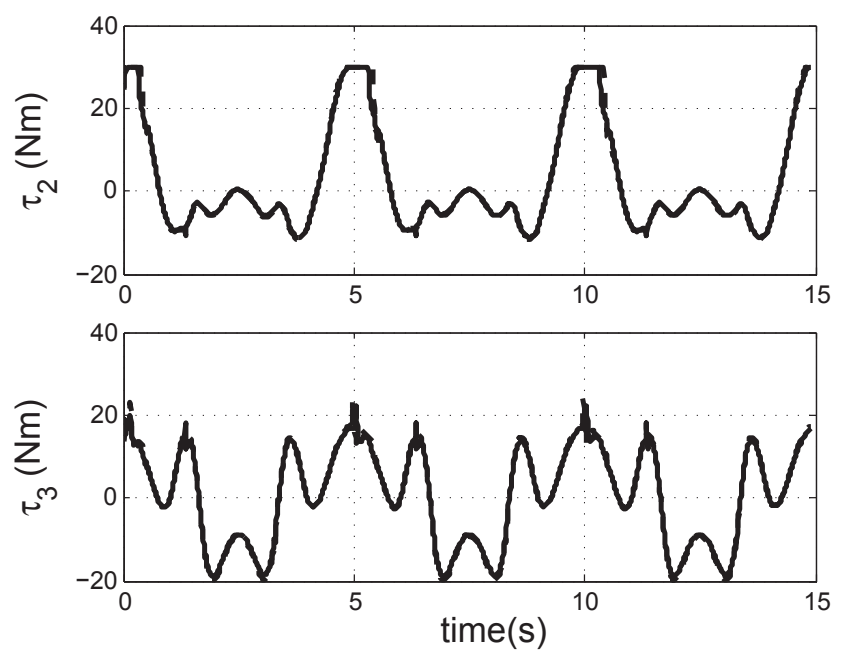

Fig. 3: Comparison of control torques for $q_{2}$ and $q_{3}$. Solid line for payload of $1.5 \mathrm{~kg}$. Dashed line for payload of $1.7 \mathrm{~kg}$.

Cost functions (1) are shown in Fig. 4 for a complete period (between 5 and $10 \mathrm{~s}$ ) and for the $1.5 \mathrm{~kg}$ payload. It also presents the changes of the controller parameter $\theta$ every time the condition (3) is satisfied by a controller of the set with better performance and the switching algorithm replaces the current controller in the loop.

Performance of UAC for joints 2 to 3 is presented in Table $\mathrm{I}$, using the ISE and CE for a time span of $15 \mathrm{~s}$ and an integration window of $5 \mathrm{~s}$. Normalization is based on the NO LOAD case, presented at the same table. From the Table I, it may be concluded that:

- The ISE for the NO LOAD case stays low and with small standard deviation.

- The ISE stays bounded for both payload values, but there is a performance loss due to the increased

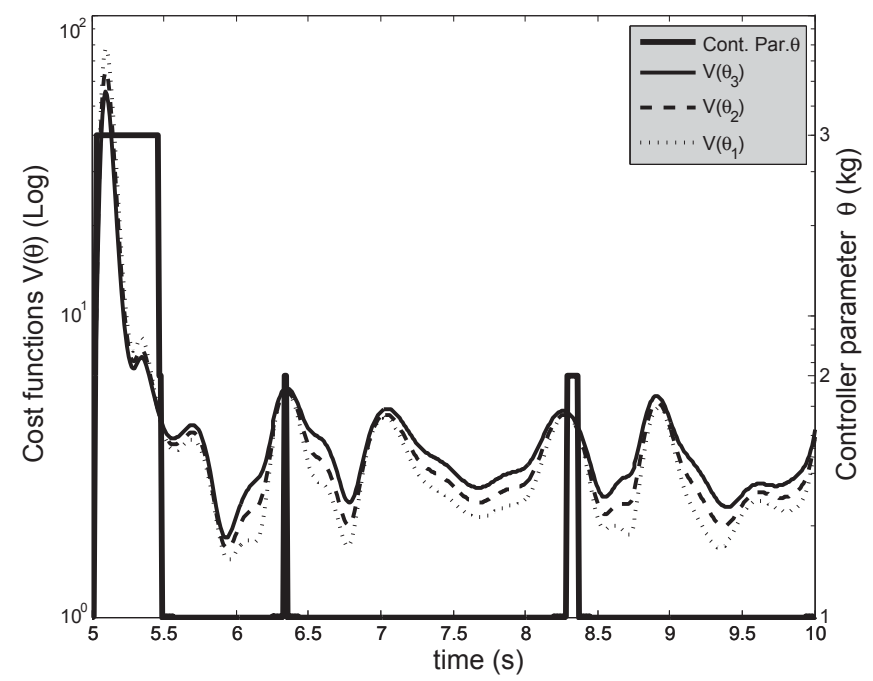

Fig. 4: UAC procedure evaluates cost functions and changes the controller parameter, for a fixed load of $1.5 \mathrm{~kg}$.

TABLE I: Performance for no-load and payloads of $1.5 \mathrm{~kg}$ and 1.7 $\mathrm{kg}$. Time span of $15 \mathrm{~s}$. Normalization based on the NO LOAD case.

\begin{tabular}{|c|l|c|c|c|}
\hline \multicolumn{3}{|c|}{ CONTROLLER } & \multicolumn{3}{c|}{ UAC } \\
\hline \multicolumn{2}{|c|}{ PAYLOAD $(\mathrm{kg})$} & NO LOAD & 1.5 & 1.7 \\
\hline \multirow{3}{*}{ ISE $q_{2}$} & MAX & $1,40 \times 10^{-3}$ & 18,21 & 74,23 \\
\cline { 2 - 5 } & MEAN & $1,29 \times 10^{-3}$ & 16,72 & 70,02 \\
\cline { 2 - 5 } & STD.DEV. & $2,21 \times 10^{-5}$ & 69,98 & 372,99 \\
\hline \multirow{3}{*}{ ISE $q_{3}$} & MAX & $5,39 \times 10^{-3}$ & 13,91 & 47,55 \\
\cline { 2 - 5 } & MEAN & $5,02 \times 10^{-3}$ & 12,74 & 44,59 \\
\cline { 2 - 5 } & STD.DEV. & $7,78 \times 10^{-5}$ & 55,10 & 241,64 \\
\hline \multirow{3}{*}{ CE $q_{2}$} & MAX & $4,33 \times 10^{2}$ & 2,16 & 2,30 \\
\cline { 2 - 5 } & MEAN & $4,16 \times 10^{2}$ & 2,15 & 2,30 \\
\cline { 2 - 5 } & STD.DEV. & 6,25 & 2,33 & 2,43 \\
\hline \multirow{3}{*}{ CE $q_{3}$} & MAX & $2,43 \times 10^{2}$ & 2,85 & 3,13 \\
\cline { 2 - 5 } & MEAN & $2,40 \times 10^{2}$ & 2,81 & 3,08 \\
\cline { 2 - 5 } & STD.DEV. & 1,85 & 2,97 & 3,30 \\
\hline
\end{tabular}

payload. Despite this, the ISE remains relatively low.

- The CE increases for both payloads, but somehow at the same scale, remaining bounded due to the stability of the controller. There are no significant differences in the $\mathrm{CE}$ due to the payload change.

\section{B. Controller performance analysis for periodic non-smooth (sudden) payload changes}

In this case, the payload changes periodically (with period $T_{l}=3 \mathrm{~s}$ ) between $0.5 \mathrm{~kg}$ and $1.5 \mathrm{~kg}$ and between $0.5 \mathrm{~kg}$ and $1.7 \mathrm{~kg}$. Sudden (non-smooth) load changes can occur in manipulators for tasks like picking up objects from shelves or helping people with personal care activities, among others.

Results show similar behaviors to the presented in the previous section for cost functions and controller parameter changes for the UAC in Fig. 4, despite of the sudden payload changes. Hence, the performance of UAC is similar to the first 
TABLE II: Controller performance comparison for joints $q_{2}$ and $q_{3}$ and non-smooth change of payload every $3 \mathrm{~s}$. Normalization based on the NO LOAD case.

\begin{tabular}{|l|l|c|c|}
\hline \multicolumn{2}{|c|}{ PAYLOAD CHANGE } & $0.5-1.5 \mathrm{~kg}$ & $0.5-1.7 \mathrm{~kg}$ \\
\hline \multirow{3}{*}{ ISE $\operatorname{rad}^{2}\left(q_{2} / q_{3}\right)$} & MAX & $0,01 / 2,26$ & $0,01 / 6,84$ \\
\cline { 2 - 4 } & MEAN & $0,01 / 2,22$ & $0,01 / 6,57$ \\
\cline { 2 - 4 } & STD. DEV. & $0,09 / 17,69$ & $0,12 / 53,54$ \\
\hline \multirow{2}{*}{$\begin{array}{l}\text { CONTROL EFFORT } \\
\mathrm{Nm}^{2}\left(q_{2} / q_{3}\right)\end{array}$} & MAX & $0,12 / 0,24$ & $0,12 / 0,24$ \\
\cline { 2 - 4 } & MEAN & $0,12 / 0,23$ & $0,12 / 0,23$ \\
\cline { 2 - 4 } & STD. DEV. & $0,10 / 1,21$ & $0,10 / 1,51$ \\
\hline
\end{tabular}

case. For the case of changes between $0.5 \mathrm{~kg}$ and $1.5 \mathrm{~kg}$, similar smooth control signals, with short transients, were noted when the controller in the loop was falsified.

A performance comparison of both controllers for joints $q_{2}$ and $q_{3}$ is presented in Table II, for sudden payload changes every $3 \mathrm{~s}$ between $0.5 \mathrm{~kg}$ and $1.5 \mathrm{~kg}$ and $0.5 \mathrm{~kg}$ and $1.7 \mathrm{~kg}$, respectively. Normalization is based on the NO LOAD case presented in Table I. From the Table II, it may be concluded that both ISE and CE remain similar to those presented in the previous scenario. This means that the controller might be used in applications such as those mentioned above, without presenting a significant loss of performance.

\section{Performance analysis as a function of the payload: from the no-load condition to the maximum of the payload interval}

Due to one performance difference between data-driven and model-based control techniques for manipulators, related to the need of a precise knowledge of its intrinsic parameters, we studied the robustness of the UAC applying disturbances (lack of precision in parameter estimation) to the inertial parameters of the model, based on the worst case scenario of parameter identification deviations of $1 \sigma$ and $2 \sigma$ confidence intervals presented by Swevers et al. [5]. This is possible by taking advantage of the model-based characteristic of the computed torque controller applied in this work for the design of the candidate controller set of the UAC and the fictitious reference computation.

Robustness was analyzed for the range between no-load and the maximum load proposed for the case of study (payload from 0 to $2 \mathrm{~kg}$ ), with resolution of $0.1 \mathrm{~kg}$, and considering worst-case model disturbances of $1 \sigma$ and $2 \sigma$ confidence intervals for the estimated inertial parameters. Results showed that the behavior from $0 \mathrm{~kg}$ to $1.4 \mathrm{~kg}$ is consistent and predictable from the previous cases, even for the proposed disturbances (the ISE and CE increased at the same rates as in previous scenarios). Hence, the following analysis is presented for payloads between $1.4 \mathrm{~kg}$ and $2 \mathrm{~kg}$, with a resolution of 0.2 $\mathrm{kg}$.

A performance comparison is shown in Table III, based on the ISE and CE for joint $q_{3}$ only ( $q_{3}$ as the elbow joint), given its significance in the proposed task. Normalization is based on the No-disturbance case, shown in the same table. Table III presents three cases: the no-disturbance case (supposing perfect knowledge of inertial parameters), the cases of $1 \sigma$ disturbance and the $2 \sigma$ disturbance of the inertial parameters. From the Table III, it may be concluded that:
TABLE III: Performance comparison using ISE and CE, for joint 3 $\left(q_{3}\right)$. Normalization based on the No-disturbance case.

\begin{tabular}{|c|c|c|}
\hline \multicolumn{3}{|c|}{ No-disturbance to the model inertial parameters } \\
\hline PAYLOAD $(\mathrm{kg})$ & ISE & CE \\
\hline 1,4 & $1,41 \times 10^{-2}$ & $6,31 \times 10^{2}$ \\
\hline 1,6 & $1,34 \times 10^{-1}$ & $7,00 \times 10^{2}$ \\
\hline 1,8 & $3,25 \times 10^{-1}$ & $7,73 \times 10^{2}$ \\
\hline 2 & $5,49 \times 10^{-1}$ & $8,53 \times 10^{2}$ \\
\hline
\end{tabular}

\begin{tabular}{|c|c|c|}
\hline \multicolumn{3}{|c|}{ Disturbance of $1 \sigma$ to the model inertial parameters } \\
\hline PAYLOAD $(\mathrm{kg})$ & ISE & CE \\
\hline 1,4 & 0,2 & 0,99 \\
\hline 1,6 & 0,6 & 0,99 \\
\hline 1,8 & 0,8 & 0,98 \\
\hline 2 & 0,9 & 0,98 \\
\hline
\end{tabular}

\begin{tabular}{|c|c|c|}
\hline \multicolumn{3}{|c|}{ Disturbance of $2 \sigma$ to the model inertial parameters } \\
\hline PAYLOAD $(\mathrm{kg})$ & ISE & CE \\
\hline 1,4 & 0,04 & 0,98 \\
\hline 1,6 & 0,54 & 0,98 \\
\hline 1,8 & 0,75 & 0,98 \\
\hline 2 & 0,84 & 0,97 \\
\hline
\end{tabular}

- In the no-disturbance case, the ISE and the CE present a bounded tendency, showing its ability to handle the higher loads of the payload testing interval with acceptable performance.

- In the case of $1 \sigma$ and $2 \sigma$ parameter disturbances, the behavior is relatively similar to the no-disturbance case and remains consistent through the entire range. The performance of the controller remains acceptable in the entire interval.

- Therefore, UAC shows independence to this kind of disturbances and then a robustness characteristic, as expected. The UAC presents a satisfactory behavior for the complete load testing interval.

\section{CONCLUSIONS}

The UAC strategy for MDoF manipulators adapts the controller parameters to seek the best performance, based exclusively on the input-output data. This condition makes the data-driven controller independent of modeling errors. Simulation results for the 5-DoF manipulator revealed the advantages of the UAC for higher loads of the testing interval. These results have also shown that the proposed controller is robust against perturbations to system parameters. UAC has also achieved a proper tracking with smooth control signals with short transients and bounded control efforts for the higher loads of the testing interval. This shows a promising chance to use this controller for different kind of MDoF manipulators, including different cases of payload handling. For future work, it is considered the evaluation of the UAC for different kind of candidate controllers and the application of the strategy 
for compliant manipulators like the WAM arm in common Human-Robot Interactive tasks like pick and place and lift and lower payloads.

\section{ACKNOWLEDGMENTS}

The authors thank the Institut de Robòtica i Informàtica Industrial (CSIC-UPC) at Universitat Politècnica de Catalunya - Barcelona Tech; the Electrical, Electronic and Computer Engineering Department at the Universidad Nacional de Colombia at Manizales, the Faculty of Engineering and Architecture, the Direction of Research at Manizales (DIMA). Finally, the authors also thank the Intelligent Perception and Control and the Soft and Hard Applied Computation Research Groups at the Universidad Nacional de Colombia for all its support.

\section{REFERENCES}

[1] A. Huang and M. Chien, Adaptive control of robot manipulators. Singapore: World Scientific Publishing Co. Pte. Ltd., 2010.

[2] J. Slotine and W. Li, Applied Nonlinear Control. New Jersey: Prentice Hall, 1991.

[3] B. Siciliano, L. Sciavicco, L. Villani, and G. Oriolo, Robotics: Modelling, Planning and Control. London: Springer Verlag, 2009.

[4] M. F. Khan, R. ul Islam, and J. Iqbal, "Control strategies for robotic manipulators," 2012 Int. Conf. Robot. Artif. Intell., pp. 26-33, Oct. 2012.

[5] J. Swevers, C. Ganseman, D. Bilgin, J. De Schutter, and H. Van Brussel, "Optimal Robot Excitation and Identification," IEEE Trans. Robot. Autom., vol. 13, pp. 730-740, 1997.

[6] J. Wu, J. Wang, and and Z. You, "An overview of dynamic parameter identification of robots," Robot. Comput. Integr. Manuf., vol. 26, pp. 414-419, 2010

[7] J. Swevers, W. Verdonck, and J. D. Schutter, "Dynamic model identification for industrial robotsIntegrated experiment design and parameter estimation," IEEE Control Syst. Mag., vol. 27, pp. 58-71, 2007.

[8] J. Hollerbach, W. Khalil, and M. Gautier, "Model identification," in Springer Handb. Robot., B. Siciliano and O. Kathib, Eds. New York: Springer-Verlag, 2008.

[9] M. Korayem, F. Davarpanah, H. Ghariblu, A. Basu, and M. H. K. F. D. H. Ghariblu, "Load carrying capacity of flexible joint manipulators with feedback linearization," Int. J. Adv. Manuf. Technol., vol. 29, no. 3-4, pp. 389-397, Mar. 2006.

[10] M. Korayem, M. Irani, and S. Rafee Nekoo, "Load maximization of flexible joint mechanical manipulator using nonlinear optimal controller," Acta Astronaut., vol. 69, no. 7-8, pp. 458-469, Sep. 2011.

[11] M. Korayem and M. Bamdad, "Analytical design of optimal trajectory with dynamic load-carrying capacity for cable-suspended manipulator," Int. J. Adv. Manuf. Technol., vol. 60, pp. 317-327, 2012.

[12] M. Safonov and T. Tsao, "The unfalsified control concept and learning," IEEE Trans. Automat. Contr., vol. 42, no. 6, pp. 843-847, Jun. 1997.

[13] M. Safonov, "Data-driven robust control design: Unfalsified Control," in Achiev. Success. Robust Integr. Control Syst. Des. 21st Century Mil. Appl. Part II, NATO, 2006, pp. 4-18.

[14] M. Stefanovic and M. G. Safonov, Lecture Notes in Control and Information Sciences 405: Safe Adaptive Control, M. Thoma, F. Allgöwer, and M. Morari, Eds. London: Springer-Verlag, 2011.
[15] T. Tsao and M. Safonov, "Unfalsified Direct Adaptive Control of a Two-Link Robot Arm," in Int. Conf. Control Appl., 1999, pp. 680-686.

[16] S. Baldi, G. Battistelli, E. Mosca, and P. Tesi, "Multi-model unfalsified adaptive switching supervisory control," Automatica, vol. 46, no. 2, pp. 249-259, Feb. 2010.

[17] G. Battistelli, E. Mosca, M. Safonov, and P. Tesi, "Stability of unfalsified adaptive switching control," IEEE Trans. Aut. Contr., vol. 55, no. 10 , pp. 2424-2429, 2010.

[18] G. Battistelli, D. Mari, D. Selvi, and P. Tesi, "Unfalsified Approach to Data-Driven Control Design," in 53rd IEEE Conf. Decis. Control, Los Angeles, California, USA, 2014, pp. 6003-6008.

[19] M. Pawluk and K. Arent, "Unfalsified control of manipulators: simulation analysis," Bull. Polish Acad. Sci. Tech. Sci., vol. 53, no. 1, pp. 19-29, 2005.

[20] F. D. Bianchi, C. Ocampo-Martinez, C. Kunusch, and R. S. SanchezPeña, "Fault-Tolerant Unfalsified Control for PEM Fuel Cell Systems," IEEE Trans. Energy Convers., vol. 30, no. 1, pp. 307-315, 2015.

[21] G. Battistelli, D. Mari, A. Riccardi, and P. Tesi, "Self-Tuning Mechanism for the Design of Adaptive Secondary Mirror Position Control," IEEE Trans. Control Syst. Technol., pp. 1-14, 2015.

[22] Y. Han, M. G. Safonov, and J. Chen, "An In-Motion Alignment Method for Inertial Navigation System Based on Unfalsified Adaptive Control Theory," in Proc. 2014 IEEE Chinese Guid. Navig. Control Conf., no. 1, Yantai, China, 2014, pp. 1900-1905.

[23] H. Jin, H. B. Siahaan, M. G. Safonov, and L.-f. Yuan, "DataDriven Switched Control for Pressure Regulation in Managed Pressure Drilling," in 32nd Chinese Control Conf. July, Xi'an, China, 2013, pp. 7906-7911.

[24] H. Jin, H. B. Siahaan, and M. G. Safonov, "Detecting Oscillation with Unfalsified Adaptive Control and Its Application in Managed Pressure Drilling for Oil," in 53rd IEEE Conf. Decis. Control, vol. 2, Los Angeles, California, USA, 2014, pp. 1271-1276.

[25] J. Wang, Y. Wand, L. Cao, and Q. Jin, "Adaptive Iterative Learning Control Based on Unfalsified Strategy for Chylla-Haase Reactor," IEEE/CAA J. Autom. Sin., vol. 1, no. 4, pp. 347-360, 2014.

[26] L. Cui, Y. X. Lv, and G. Xie, "Unfalsified control research and its application in inverted pendulum system," in 25th Chinese Control Decis. Conf., 2013, pp. 2259-2263.

[27] H. Jin, M. W. Chang, and M. G. Safonov, "A fading memory data-driven algorithm for controller switching," in IEEE Conf. Decis. Control Eur. Control Conf. Orlando, FL: IEEE, 2011, pp. 6097-6103.

[28] K. R. Popper, Conjectures and Refutations: The Growth of Scientific Knowledge. London: Routhledge, 1963.

[29] T. Tsao and M. G. Safonov, "Unfalsified Direct Adaptive Control of a Two Link Robot Arm," in Proc. IEEE Conf. Control Appl., Hawai (USA), 1999, pp. 680-686.

[30] A. S. Morse, D. Q. Mayne, and G. C. Goodwin, "Applications of hysteresis switching in parameter adaptive control," IEEE Trans. Autom. Control, vol. 37, no. 9, pp. 1343--1354, 1992.

[31] S. Durango and M. Calderon, "Kinematical, dynamical and manipulability model of the Scorbot ER V PLUS manipulator robot," Master's thesis, Universidad Tecnológica de Pereira, 2006.

[32] J. Arango, "Dynamical analysis and nonlinear control of a 5-DoF manipulator," Master's thesis, Universidad Nacional de Colombia, 2008.

[33] R. Wang and M. Safonov, "Stability of unfalsified adaptive control using multiple controllers," Proc. 2005, Am. Control Conf. 2005., pp. 31623167, 2005. 